\title{
Evaluation du statut nutritionnel et biologique d'un échantillon de personnes atteintes de glaucome à angle ouvert dans une population togolaise
}

\author{
Essodolom PAKA ${ }^{1 *}$, Idès BILABINA ${ }^{1}$, Mamatchi MELILA ${ }^{1}$, Abiba BANLA-KERE ${ }^{3}$, \\ Mèba BANLA ${ }^{2}$, Wiyao POUTOULI ${ }^{4}$ et Kou'santa AMOUZOU ${ }^{1}$ \\ ${ }^{1}$ Laboratoire de Biochimie/Nutrition, Faculté des Sciences, Université de Lomé, Togo. \\ ${ }^{2}$ Laboratoires du Service ophtalmologie du CHU Campus de Lomé, Togo. \\ ${ }^{3}$ Laboratoire de l'Institut National d'Hygiène de Lomé, Togo. \\ ${ }^{4}$ Laboratoire de Biologie Animale et de Zoologie, Faculté des Sciences, Université de Lomé, Togo. \\ *Auteur correspondant, E-mail : pakeslome@yahoo.fr, Tél : (00228) 90147862/99556782
}

\section{RESUME}

Ce travail est une étude prospective menée d'octobre 2009 à juillet 2010. L'objectif a été d'évaluer dans un échantillon de population togolaise les facteurs biologiques et nutritionnels des patients atteints de glaucome à angle ouvert. L'étude a porté sur deux groupes d'individus des deux sexes : 35 patients ayant un glaucome à angle ouvert et 27 témoins. L'âge, le poids, la taille et l'IMC moyens ont été similaires entre les deux groupes. Les sujets ont tous subi le même bilan biologique. Le t-test a été utilisé pour l'évaluation statistique. Les résultats ont révélé que chez les patients, les taux moyens de glycémie, de cholestérol total, des triglycérides, de HDL-cholestérol et de LDL-cholestérol ont été plus élevés $(\mathrm{p}<0,001)$ comparativement à ceux des témoins. Le taux moyen de la créatinine chez les patients a été similaire à celui des témoins. Il en est de même pour les taux moyens de la protidémie. En ce qui concerne les transaminases et la GGT, les taux moyens chez les patients ont été plus élevés que chez les témoins $(\mathrm{p}<0,05)$. Il ressort de ces résultats que la glycémie, le cholestérol total, les HDL, les LDL, les triglycérides, les transaminases et la GGT pourraient être inclus dans les facteurs de risque du glaucome à angle ouvert. L'augmentation du taux de ces différents paramètres serait probablement d'origine alimentaire.

(C) 2014 International Formulae Group. All rights reserved.

Mots clés : Glaucome à angle ouvert, alimentation, bilan biologique.

\section{INTRODUCTION}

Le glaucome chronique à angle ouvert est une pathologie caractérisée par une atteinte du nerf optique, du champ visuel et un angle irido-cornéen ouvert. Il est associé en général à une pression intraoculaire caractéristique élevée. Aux côtés des autres facteurs de risque (âge, hypertension artérielle, diabète, antécédents familiaux), une pression intraoculaire élevée constitue le facteur de risque majeur du glaucome à angle ouvert (Sommer et al., 1991). Mais la définition du glaucome comme pression intraoculaire élevée entraînant une perte de vision a progressivement évoluée. Aujourd'hui le glaucome, au-delà d'une maladie de l'œil, est considéré comme une affection dégénérative du système nerveux 
similaire à la maladie d'Alzheimer ou celle du Parkinson (Ahnoux-Zabsonre et al., 1998). Ces conditions dégénératives affectent une population âgée et impliquent une perte sélective de certains neurones (Sommer et al., 1991).

Malgré les efforts entrepris pour éradiquer cette affection, elle reste une cause majeure de malvoyance et de cécité dans le monde (Mac donald, 1965; Olurin, 1970 ; Lcheie, 1965). Le glaucome à angle ouvert représente 80 à $90 \%$ des cas de glaucome. Des travaux menés en Afrique ont par ailleurs relevé certaines particularités du glaucome à angle ouvert intertropical (Newman et Zanberman, 1965).

L'objectif de cette étude a été d'évaluer dans un échantillon de la population togolaise les facteurs biologiques et nutritionnels des patients présentant un glaucome à angle ouvert. Ainsi, il a été réalisé des analyses biochimiques de certains facteurs de risques, afin de présenter un profil biologique et nutritionnel de ces patients atteints de glaucome à angle ouvert.

\section{MATERIEL ET METHODES}

\section{Cadre de l'étude}

L'étude a été réalisée au Centre Hospitalier Universitaire Campus (CHUCampus) de Lomé et à l'Institut National d'Hygiène (INH) de Lomé (Sud-Togo). Le recrutement des patients a été effectué dans le service d'ophtalmologie du CHU-Campus de Lomé lors des consultations. Ensuite, ces patients sont envoyés à l'INH pour des analyses biologiques.

L'étude a porté sur deux groupes d'individus des deux sexes : 35 patients dont 20 hommes $(57,2 \%)$ et 15 femmes $(42,8 \%)$ souffrant de glaucome à angle ouvert et 27 témoins regroupant 19 hommes $(70,4 \%)$ et 8 femmes $(29,6 \%)$, soit au total 62 sujets.

\section{Population cible \\ Critères d'inclusion}

Les patients ayant fait l'objet de cette étude ont été des sujets qui ont bénéficié des examens cliniques ou qui ont des signes relatifs au glaucome à angle ouvert notamment :

- une altération du champ visuel,

- une pression intraoculaire mesurée à l'aplanation de Goldman,

- une pachymétrie pour mesurer l'épaisseur centrale de la cornée et corriger la valeur de la pression intraoculaire,

- un fond de l'œil pour rechercher l'excavation papillaire caractéristique,

- une gonoscopie pour s'assurer de l'ouverture complète de l'angle,

- une sélection aléatoire sans tenir compte du sexe et de l'âge.

Les témoins ont été des sujets n'ayant aucun signe relatif au glaucome à angle ouvert et aucun antécédent par rapport au glaucome dans la famille.

\section{Prélèvement du sang}

Les prélèvements sanguins ont été effectués sur tous les sujets à jeun, dans des tubes secs et des tubes EDTA, les matins de 7 $h$ à 9 h à l'INH. Les conditions de prélèvement et de conservation des échantillons ont été standardisées pour permettre une interprétation de l'évaluation plasmatique de différents paramètres biochimiques. Les échantillons ont été conservés à l'INH dans un réfrigérateur de type R P1190 C à une température comprise entre 0 et $2{ }^{\circ} \mathrm{C}$. Dans le souci d'éviter d'autres réactions après prélèvement et lors de la conservation, nous avons utilisé des tubes EDTA (anticoagulant) et ensuite nous avons entrepris une séparation rapide des hématies du plasma, dans un délai inférieur à 30 minutes à la température ambiante.

\section{Paramètres anthropométriques étudiés}

A partir des fiches d'enquête de consommation alimentaire, nous avons recueilli des données sur l'âge, et le sexe de chaque sujet. Aussi, il a été déterminé la taille et le poids qui ont permis de calculer l'indice de masse corporelle (IMC) pour chaque sujet. 


\section{Paramètres biochimiques étudiés}

Les analyses ont porté sur les paramètres suivants : Glycémie, Créatinémie, Cholestérol Total, HDL-Cholestérol, LDLCholestérol, Triglycérides, Aspartate aminotransférase (ASAT), Alanine aminotransférase (ALAT), Gammaglytamyltransférase (GGT), Protidémie.

\section{Méthodes analytiques}

Le dosage du glucose a été effectué selon la méthode de Trinder (1969). Le dosage de la créatinine a été réalisé selon un procédé automatisé par la méthode Jaffé (Fabiny et Ertingshausen, 1971). Les activités enzymatiques d'ASAT et d'ALAT ont été déterminées par la méthode optimisée de Henry (1960) et celle de GGT par la technique de Szasz (1969). Le dosage du cholestérol total a été réalisé selon la méthode enzymatique décrite par Allain (1974). Les concentrations de HDL et de LDL ont été déterminées selon la méthode de Naito (1984). Le taux des triglycérides a été déterminé par la méthode de Fossati, 1982) couplée à la réaction de Trinder (1969). Les protéines totales ont été dosées par la méthode colorimétrique de Biuret (Burtis et Tietz, 1999).

\section{Analyse statistique}

Les données ont été traitées statistiquement à l'aide du programme Microsoft Excel de Windows 2007. Le t-test et le calcul des moindres différences significatives de Fisher ont servi pour la comparaison des données. Les différences ont été considérées comme significatives à un seuil de $5 \%$.

\section{RESULTATS}

Les données anthropométriques (Poids, Taille, Sexe, IMC) des sujets ayant fait l'objet de cette étude sont consignées dans le Tableau 1. Ces données montrent que l'âge moyen en années des patients et celui des témoins ne sont pas significativement différents. Il en est de même pour le poids et la taille. Aussi,
l'IMC moyen des patients ne diffère pas significativement de celui des témoins.

Les informations suivantes ont également été enregistrées:

- 14,28\% des patients sont diabétiques contre $0 \%$ chez les témoins ;

- 28,57\% des patients sont porteurs du virus de l'hépatite $\mathrm{B}$ contre $7,4 \%$ chez les témoins.

La répartition des sujets en fonction de l'index glycémique (IG) est indiquée dans le Tableau 2. Ce critère de classement des glucides permet de définir le pouvoir d'un aliment à augmenter le taux de glucose dans le sang. Le Tableau 2 montre que :

- $35 \%$ des patients contre $27 \%$ des témoins ont un index glycémique élevé $(\geq 70)$. Ces sujets consomment régulièrement des aliments tels que : sucre, riz à cuisson rapide, patate douce, carottes cuites, pommes de terre. - $25 \%$ des patients contre $18 \%$ des témoins ont présenté un index glycémique moyen (entre 56 et 69). Ces personnes ont l'habitude de consommer des aliments tels que : couscous, pâtes de maïs, banane mûre, mayonnaise.

- $40 \%$ des patients contre $55 \%$ des témoins ont été enregistrés dans le cas d'un index glycémique faible (entre 0 et 55). Les aliments habituellement consommés par ces sujets sont: mangue; jus de pamplemousse, d'orange et de citron sans sucre; tomate; banane verte ; orange ; pêche ; yaourt naturel ; lait ; ail ; carottes crues ; aubergine ; choux ; oignon ; épices.

Les résultats ont révélé aussi que presque tous les sujets consomment des graisses animales et huiles végétales; $35 \%$ des patients contre $38 \%$ des témoins préfèrent des fruits et des légumes ; $70 \%$ des patients prennent régulièrement de l'alcool contre $50 \%$ des témoins.

La Figure 1 présente les taux de glycémie, de créatinine et de protidémie des sujets. Ces résultats montrent que chez les patients, le taux moyen de glycémie $(1,35 \pm$ $0,73 \mathrm{~g} / \mathrm{l})$ est plus élevé comparativement à celui des témoins estimé à $0,85 \pm 0,19 \mathrm{~g} / \mathrm{l}(\mathrm{p}<$ 
0,001). Par contre, le taux moyen de la créatinine chez les patients $(10,11 \pm 2,42$ $\mathrm{mg} / \mathrm{l})$ ne diffère pas significativement de celui observé chez les témoins évalué à 10,41 \pm $2,36 \mathrm{~g} / \mathrm{l}$. Il en est de même pour les taux moyens de protidémie qui sont de $71,71 \pm$ $3,36 \mathrm{~g} / \mathrm{l}$ et de $72,0 \pm 3,60 \mathrm{~g} / \mathrm{l}$ respectivement chez les patients et les témoins.

Chez les patients, les taux moyens de cholestérol total $(1,95 \pm 0,33 \mathrm{~g} / \mathrm{l})$, des triglycérides $(1,22 \pm 0,27 \mathrm{~g} / \mathrm{l})$, de HDLcholestérol $(0,46 \pm 0,07 \mathrm{~g} / \mathrm{l})$ et de LDLcholestérol $(1,32 \pm 0,31 \mathrm{~g} / \mathrm{l})$ diffèrent significativement $(\mathrm{p}<0,001)$ de ceux des témoins estimés à $1,59 \pm 0,39 \mathrm{~g} / \mathrm{l}$, pour le cholestérol total, $0,73 \pm 0,28 \mathrm{~g} / \mathrm{l}$ pour les triglycérides, $0,36 \pm 0,09 \mathrm{~g} / \mathrm{l}$ pour le HDLcholestérol et $1,08 \pm 0,35 \mathrm{~g} / \mathrm{l}$ pour le LDLcholestérol (Figure 2).

La Figure 3 illustre les taux moyens d'ASAT, d'ALAT et de GGT. Ces taux chez les patients sont respectivement de 38,65 $\mathrm{UI} / \mathrm{L}$; 34,88 UI/L et 39,06 UI/L, tandis que chez les témoins ces taux sont de 27,04 UI/L pour l'ASAT ; 25,00 UI/L pour l'ALAT et 23,41 UI/L pour la GGT. La différence observée a été significative $(\mathrm{p}<0,05)$.

Tableau 1 : Paramètres anthropométriques.

\begin{tabular}{lccccc}
\hline Sujets & Nombre de sujets & Age $(\mathbf{a n s})$ & Poids $\mathbf{( k g )}$ & Taille $(\mathbf{m})$ & IMC $\left(\mathbf{k g} / \mathbf{m}^{\mathbf{2}}\right)$ \\
\hline Patients & 35 & $42,66 \pm 5,10$ & $77,46 \pm 12,01$ & $1,70 \pm 0,06$ & $26,46 \pm 3,46$ \\
Témoins & 27 & $41,63 \pm 14,56$ & $77,74 \pm 13,64$ & $1,68 \pm 0,07$ & $25,63 \pm 3,62$ \\
P value & $>0,05$ & $>0,05$ & $>0,05$ & $>0,05$ & $>0,05$ \\
\hline
\end{tabular}

Tableau 2 : Répartition des sujets en fonction de l'index glycémique (IG).

\begin{tabular}{lccc}
\hline Sujets & \multicolumn{3}{c}{ Index glycémique } \\
\cline { 2 - 4 } & $\mathbf{0}$ à 55 & $\mathbf{5 6}$ à 69 & $\geq \mathbf{7 0}$ \\
\hline Patients & $40 \%$ & $25 \%$ & $35 \%$ \\
Témoins & $55 \%$ & $18 \%$ & $27 \%$ \\
\hline
\end{tabular}

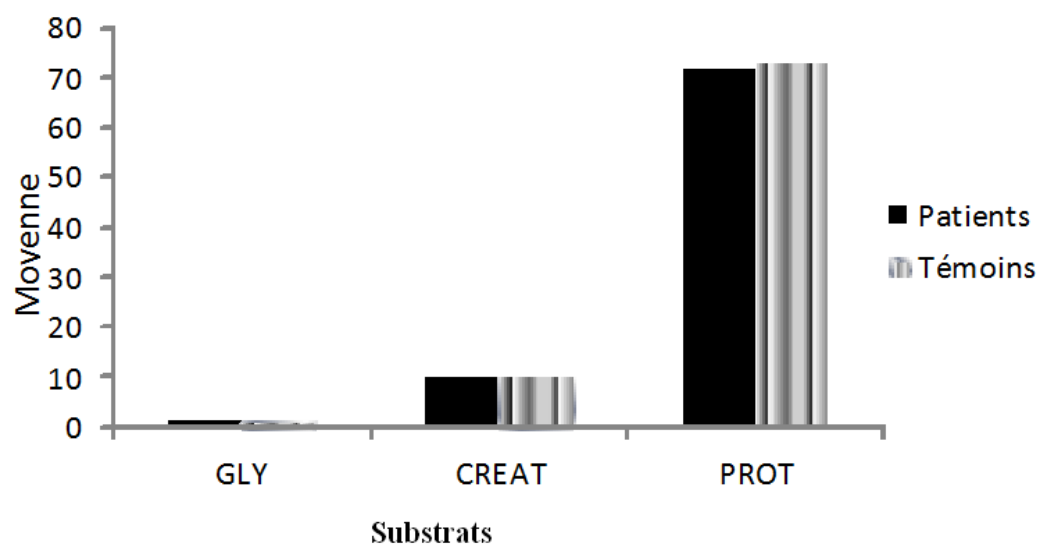

Figure 1: Taux de glycémie, de créatinine et de protidémie chez les sujets. GLY = glycémie; CREAT $=$ créatinine $;$ PROT $=$ protidémie . 


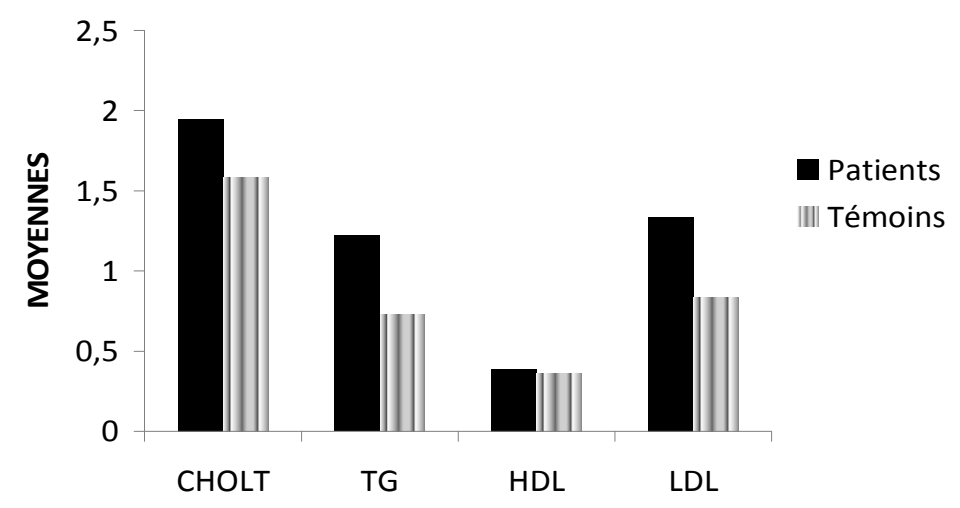

Figure 2: Taux de cholestérol total, de triglycérides; de HDL et de LDL chez les sujets. $\mathrm{CHOLT}=$ cholestérol total; $\mathrm{TG}=$ triglycérides $; \mathrm{HDL}=$ high density lipoprotein $; \mathrm{LDL}=$ low density lipoprotein.

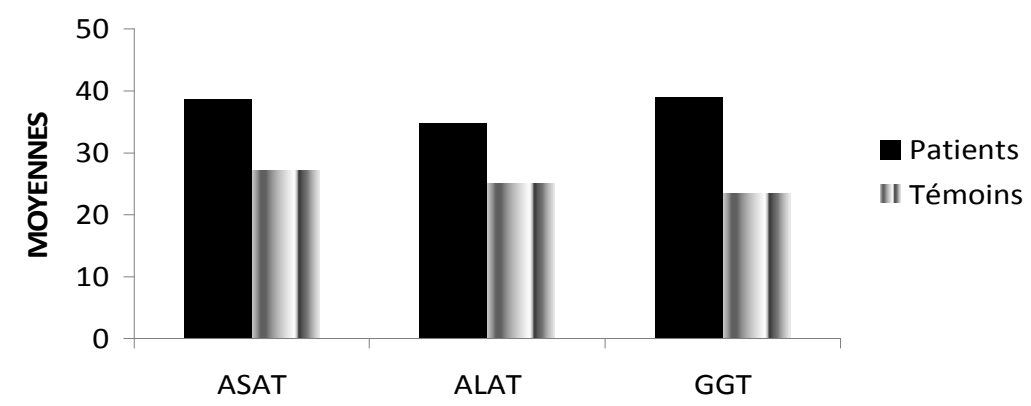

Figure 3 : Taux de transaminases et de gamma-glutamyltransférase chez les sujets. ASAT \& ALAT = transaminases ; GGT = gamma-glutamyltransférase.

\section{DISCUSSION}

Les résultats de cette étude ont montré que l'âge, le poids et la taille moyens des patients et des témoins n'ont pas été significativement différents. Il a été de même pour l'IMC. Cependant, en se référant à la norme de l'indice de masse corporelle (18,5 à $24,9)$, on peut conclure que les sujets enregistrés pour cette étude présentent une surcharge pondérale (IMC > 25). Il a été montré que le surpoids entraîne l'obésité, et par conséquent le diabète de type 2 , qui est en augmentation constante dans les pays en voie de développement du fait d'une consommation calorique excessive et d'une moindre dépense énergétique. Ce type de diabète est associé à un risque accru de glaucome primaire à angle ouvert (Louis, 2006). Le sexe masculin semble être également un facteur de risque de cette pathologie (Bachétoille, 1987 ; Leske et al., 1995). Dans notre étude, les hommes représentent $57,14 \%$ des patients. Des études antérieures en Côte d'Ivoire (AhnouxZabsonre et al., 1998), à Tunis (Ouertani et al., 1995) de même qu'au Togo (Balo et Talabe, 1994), ont aussi montré une prédominance masculine dans le cas de cette 
pathologie. Le taux moyen de glycémie chez les patients $(1,35 \pm 0,73 \mathrm{~g} / \mathrm{l})$ est plus élevé comparativement à celui des témoins estimé à $0,85 \pm 0,19 \mathrm{~g} / \mathrm{l}(\mathrm{p}<0,001)$. La glycémie serait donc probablement un facteur de risque dans le développement du glaucome à angle ouvert tout comme le diabète. Les résultats de cette étude ont révélé que $35 \%$ des patients contre $27 \%$ des témoins avaient un index glycémique élevé ( $\geq 70$ ) parce qu'ils consomment particulièrement des aliments riches en glucides tels que : sucre, riz à cuisson rapide, patate douce, carottes cuites, pommes de terre.

L'index glycémique est un critère qui permet de définir le pouvoir d'un aliment à augmenter le taux de glucose dans le sang. Plus un glucide est assimilable rapidement dans le sang, plus son index glycémique est élevé. De ce fait, il ne procure alors aucune sensation de satiété, mais provoque une sécrétion brutale d'insuline et facilite ainsi, le stockage des graisses dans les cellules. Au contraire, les aliments à faible index glycémique sont un bon moyen de contrôler sa faim, de réguler ses taux d'insuline et de brûler les graisses sans diminuer la masse musculaire. Le taux moyen de créatinine chez les patients $(10,11 \pm 2,42 \mathrm{mg} / \mathrm{l})$ ne diffère pas significativement de celui observé chez les témoins évalué à $10,41 \pm 2,36 \mathrm{~g} / \mathrm{l}$. Ces résultats corroborent ceux de Froussart et al. (2003) dont les travaux ont porté sur 53 patients glaucomateux et 46 témoins. On pourrait ainsi conclure qu'il n'aurait pas de corrélation entre la créatinine et le risque accru de glaucome. La quantité de créatinine produite chaque jour est fonction de la masse musculaire, de l'âge, du sexe, de l'alimentation ou de l'exercice physique et varie peu d'un jour à l'autre (Tietz, 1999). Le taux moyen de protidémie des patients et celui des témoins ne diffèrent pas aussi significativement. Ce résultat infirme celui de Froussart et al. (2003) qui ont obtenu des taux d'albumine sérique et de protidémie plus élevées chez les patients glaucomateux par rapport aux témoins. Ces différences peuvent s'expliquer par le fait que les deux études n'ont pas été menées dans les mêmes conditions. Les habitudes alimentaires pourraient également en partie l'expliquer. En outre, certains patients ne souffrent pas seulement de maux d'yeux et cela pourrait beaucoup influencer sur les paramètres biologiques tels que la protidémie (Burtis et Tietz, 1999). Les taux de dyslipidémie ont montré que chez les patients, les taux moyens des triglycérides, de cholestérol total, de HDL-cholestérol et de LDL-cholestérol diffèrent significativement $(\mathrm{p}<0,001)$ de ceux des témoins. Ces résultats sont en accord avec ceux obtenus par Pavljasević et Asćerić (2009). Les dyslipidémies constitueraient donc un facteur de risque dans le développement du glaucome à angle ouvert. Les taux des dyslipidémies peuvent être influencés par certains facteurs nutritionnels. Ces facteurs, d'une façon, élèvent la cholestérolémie essentiellement à partir des acides gras saturées (AGS) ou d'une autre, abaissent la cholestérolémie par la consommation des acides gras insaturés (AGI). Les acides gras polyinsaturés de la série (n-3) ont potentiellement un effet antiinflammatoire (au niveau artériel) et antithrombotique. Nous avons noté une prévalence élevée des transaminases et de la gamma-glutamyltransférase dans le risque accru du glaucome à angle ouvert. Les données de cette étude ont révélé en effet que les taux moyens d'ASAT, d'ALAT et de la GGT chez les patients ont été respectivement de $38,65 \mathrm{UI} / \mathrm{L}, 34,88 \mathrm{UI} / \mathrm{L}$ et $39,06 \mathrm{UI} / \mathrm{L}$, tandis que chez les témoins, ces taux ont été de 27,04 UI/L pour l'ASAT, 25,0 UI/L pour l'ALAT et 23,41 UI/L pour la GGT avec des différences significatives $(\mathrm{p}<0,05)$. Cependant, cette étude doit être approfondie pour une meilleure conclusion du fait que certains de nos patients ont témoigné avoir eu 
certains problèmes hépatiques qui pourraient influencer les taux de ces enzymes.

\section{Conclusion}

Il se dégage de ce travail que la glycémie, le cholestérol total, les HDL, les LDL, les triglycérides, les transaminases (ASAT et ALAT) et la gammaglutamyltransférase pourraient être considérés, à des degrés divers, comme des facteurs de risque dans le développement du glaucome à angle ouvert. De ce fait, la consommation de l'alcool et du tabac qui pourraient diminuer l'activité $\mathrm{du}$ foie favorisant l'élévation du taux des transaminases et de la GGT devra être déconseillée. Aussi, des précautions pour éviter certaines maladies hépatiques telles que les hépatites $\mathrm{B}$ et $\mathrm{C}$ pourraient diminuer le glaucome à angle ouvert. L'attention de nos populations doit être attirée pour l'adoption des habitudes alimentaires en vue d'augmenter les teneurs en HDL protecteur et de réduire les teneurs en LDL, en triglycérides, en cholestérol total et surtout en glucose dans le sang. Il serait souhaitable que cette étude soit menée sur un échantillon de population plus représentatif en tenant compte de l'état de santé de chaque patient, des habitudes alimentaires et de la variabilité génétique. En outre, d'autres analyses biochimiques telles que les taux de glutamate, d'homocystéine sont à entreprendre pour approfondir les connaissances sur les facteurs de risque de glaucome à angle ouvert.

\section{REFERENCES}

Ahnoux-Zabsonre A, Keita C, Safede C, Tanoe A. 1998. Prévalence du glaucome chronique primitif à angle ouvert en Côte d'Ivoire. J. Fr. Ophtalmol., 9: 643-647.

Allain C. 1974. Enzymatic Determination of Total Serum Cholesterol. Clinical Chemistry, 20: 470-475.
Balo KP, Talabe M. 1994. Les jeunes glaucomateux togolais. J. Fr. Ophtalmol., 11: 668-673.

Bechétoille A. 1987. Épidémiologie et Facteurs de Risque du Glaucome. Ed. Japperenard : Nantes, 253-255.

Burtis A, Tietz. 1999. Textbook of Clinical Chemistry ( $3^{\text {rd }}$ edn). AACC ; 652-657.

Fabiny DL, Ertingshausen G. 1971. Automated reaction-rate method for determination of serum creatinine with the CentrifiChem. Clinical Chemistry, 17: 696-700.

Fossati P, Prencipe L. 1982. Serum Triglycerides Determined Colorimetrically with an Enzyme That Produces Hydrogen Peroxide. Clinical Chemistry, 28(10): 2077-2080.

Froussart F, Renard JP, Didelot F, Ott D, Dot C, Rigal-Sastourne JC, Bidaux F, Maurin JF. 2003. Etude Des Facteurs Métaboliques dans le Glaucome Primitif à Angle Ouvert. Revue Ophtalmologie, 989-1005.

Henry RJ, Chiamori N, Golub OJ, Berkman S. 1960. Revised spectrophotometric methods for the determination of glutamic-oxalacetic transaminase, glutamic-pyruvic transaminase, and lactic acid dehydrogenase. Am J. Clin. Pathol., 34: 381-398.

Lcheie HG. 1957. Width and pigmentation of the angle of the anterior chamber. A system of grading by gonioscopy. Arch Ophthalmol., 58: 510.

Leske M, Connell A, Wu S, Hyman L, Schachat A. 1995. Risk factors for openangle glaucoma. Arch Ophthalmol., 7: 918-924.

Louis P. 2006. Le diabète de type 2 et le glaucome à angle ouvert. Ophthalmology, 113(7): 1081-1086.

Naito HK. 1984. High-density lipoprotein (HDL) cholesterol. The C.V. Mosby Co. St Louis. Toronto. Princeton, 1207-1213. 
Newman E et Zanberman H. 1965. Glaucoma survey in Liberia. Amer. J. Opht., 59: 812.

Olurin O. 1970. Etiology of blindness in Nigerian children. Amer. J. Opht., 70(4): 533-540.

Ouertani A, Zhioua R, Trabelsi A, Jrad J. 1995. Prévalence du glaucome chronique à angle ouvert dans une commune de Tunis. J. Fr. Ophtalmol., 3: 178-182.

Pavljasević S, Asćerić M. 2009. Primary open-angle glaucoma and serum lipids. Bosn. J. Basic. Med. Sci., 9(1): 85-88.

Sommer A, Tielsch JM, Katz J. 1991. Relationship between intraocular pressure and primary open angle glaucoma among white and black americans. Arch. Ophthalmol., 109: 1090-1095.

Szasz G. 1969. Advances in clinical chemistry. Clinical Chemistry, 15 : 124.

Tietz NW. 1999. Text Book of Clinical Chemistry $\left(3^{\text {rd }}\right.$ edn). Burtis CA, Ashwood ER, Saunders WB (eds); 652-689.

Trinder P. 1969. Determination of blood glucose using 4-aminophenazone as oxygen acceptor. Annals of Clinical Biochemistry, 6: 24-27. 